\title{
Distinct Proteomic Profiling of Plasma Extracellular Vesicles from Moderate-to-Severe Atopic Dermatitis Patients
}

Chih-Jung Chang, 1,2,* Hai-Qing Wang, ${ }^{3, *}$ Jing Zhang, ${ }^{3}$ Ying Zou, ${ }^{3}$ Yi-Hua Zhang, ${ }^{3}$ Jia-Wen Chen, ${ }^{3}$ Chun-Bing Chen, (D) ${ }^{2,4-7}$ Wen-Hung Chung, $1,2,4,6,8,9$ Chao $\mathrm{ji}^{3}$

'Medical Research Center and Xiamen Chang Gung Allergology Consortium, Xiamen Chang Gung Hospital, Xiamen, Fujian, People's Republic of China; ${ }^{2}$ Department of Dermatology and Drug Hypersensitivity Clinical and Research Center, Chang Gung Memorial Hospital, Linkou, Taipei and Keelung, Taiwan; ${ }^{3}$ Department of Dermatology, The First Affiliated Hospital of Fujian Medical University, Fuzhou, Fujian, People's Republic of China; ${ }^{4}$ Cancer Vaccine and Immune Cell Therapy Core Laboratory, Department of Medical Research, Chang Gung Memorial Hospital, Taoyuan, Taiwan; ${ }^{5}$ College of Medicine, Chang Gung University, Taoyuan, Taiwan; ${ }^{6}$ Whole-Genome Research Core Laboratory of Human Diseases, Chang Gung Memorial Hospital, Keelung, Taiwan; ${ }^{7}$ Graduate Institute of Clinical Medical Sciences, Chang Gung University, Taoyuan, Taiwan;

${ }^{8}$ Department of Dermatology, Xiamen Chang Gung Hospital, Xiamen, Fujian, People's Republic of China; 'School of Medicine, Shanghai Jiao Tong University, Shanghai, People's Republic of China

*These authors contributed equally to this work

Correspondence: Wen-Hung Chung Department of Dermatology and Drug Hypersensitivity Clinical and Research Center, Chang Gung Memorial Hospital, Linkou, Taipei and Keelung, Taiwan

Tel +886-3-328I 200 ext. 8494

Email wenhungchung@yahoo.com

Chao Ji

Department of Dermatology, The First Affiliated Hospital of Fujian Medical University, Fuzhou, Fujian, People's Republic of China Tel +86-18651619908

Email jichaofy@fjmu.edu.cn
Background: Atopic dermatitis (AD) is a chronic, inflammatory cutaneous disorder characterized by a T helper 2 (Th2) immune response phenotype. Extracellular vesicles (EVs) are a heterogeneous family of cell-derived membranous structures, which transport cellular components such as DNA and proteins, and are involved in multiple physiological and pathological processes. Increasing evidence has shown that EVs secretion took part in the pathogenesis of $\mathrm{AD}$. However, the proteomic studies of plasma-derived EVs in AD patients have not been reported.

Objective: In this study, we investigated the diversity of plasma EVs collected from AD patients and healthy individuals and suggested that the candidates for uniquely or differentially expressed proteins in plasma EVs could be a diagnostic marker in AD.

Methods: The plasma EVs were collected from 12 patients with moderate-to-severe AD and 13 healthy subjects. Proteomic analysis was performed by using a comprehensive nanoLC-MS/MS method.

Results: Proteomic analysis revealed that a total of 1478 proteins in plasma EVs were found to be common proteins in $\mathrm{AD}$, whereas a total of 1597 proteins in plasma EVs were found to be common proteins in HC. Eighty-six proteins in plasma EVs showed more than 2.5-fold up-regulation, while a total of 225 proteins in plasma EVs showed less than 1/2.5-fold downregulation with a significant difference $(\mathrm{p}<0.05)$ among $\mathrm{AD}$ compared with $\mathrm{HC}$. The candidates for differentially expressed proteins in plasma EVs have been described as a connectivity PPI network related to several KEGG pathways, including pathways in platelet activation, complement, and so on.

Conclusion: SLP-76 tyrosine phosphoprotein (SLP76) involved in platelet activation may significantly contribute to the pathogenesis of AD. We will further verify the role of SLP67 in $\mathrm{AD}$ via animal and cell experiments to provide a promising therapeutic or diagnostic target.

Keywords: atopic dermatitis, extracellular vesicle, plasma, proteomic profiling

\section{Introduction}

Atopic dermatitis (AD) is a common, chronic, inflammatory cutaneous disorder presenting recurrent eczematous lesions and intense pruritus that affects children and adults. It is also characterized by a $\mathrm{T}$ helper 2 (Th2) immune response phenotype. ${ }^{1}$ AD has a substantial physical and psychosocial impact on patients and relatives. ${ }^{2,3}$ The estimated prevalence of $\mathrm{AD}$ ranges from $15 \%$ to $20 \%$ in children and $7 \%$ to $10 \%$ in adults. ${ }^{4,5}$ It is the leading cause of the global burden 
from skin disease. ${ }^{1}$ The pathophysiology of AD is multifactorial and complex, involving genetic component, dysfunction of epidermal barrier, skin microbiome and immune dysregulation. $^{2}$

Extracellular vesicles (EVs) are a heterogeneous family of cell-derived membranous structures, including exosomes and microvesicles. ${ }^{6}$ They transport cellular components such as microRNAs, mRNAs, DNA and proteins, and are involved in multiple physiological and pathological processes. It has been reported that EVs play essential roles in immune response, tumor pathogenesis, neurodegenerative pathogenesis and pathogenic infections. ${ }^{7-10}$ EVs also have the ability to serve as biomarkers, vaccine and drug carriers engineered. ${ }^{11}$ Increasing evidence has shown that EVs secretion takes part in the pathogenesis of skin diseases, such as systemic lupus erythematosus and AD. ${ }^{12}$

Proteomics is the characterization of proteome, involving expression, structure, functions, interactions and modifications of proteins in a cell, tissue or an organism. Proteomics plays a vital role in early disease diagnosis, prognosis and the development of disease. Furthermore, it is also crucial for drug development as target molecules. ${ }^{13,14}$ Although multiple sample resources are available, peripheral blood has been regarded as an ideal source due to its reproducibility of measurement and feasibility of access. ${ }^{15}$ However, $99 \%$ of serum proteins, including albumin, immunoglobulins, and complement factors, will mask some biomarker candidates when measured by conventional proteomics. To break down these barriers, we focused on proteomic profiling of EVs.

In a recent study, protein analysis of exosomes derived from human adipose tissue-derived mesenchymal stem cells (ASC-exosomes) has shown that ASC-exosomes effectively restore epidermal barrier functions in $\mathrm{AD}$ by facilitating the synthesis of ceramides. ${ }^{16}$ Cho et al $^{17}$ also prompted that ASC-exosomes could be engaged in immune regulation. Hong et $\mathrm{al}^{18}$ have revealed that EVs derived from Staphylococcus aureus could induce AD-like inflammation in the skin. Yang et $\mathrm{al}^{19}$ supported that microbial EVs-based metagenomic markers might detect the risk of $\mathrm{AD}$ development. These series of studies have shown an inextricable link between EVs and the pathophysiology of AD. However, the proteomic studies of plasma-derived EVs in $\mathrm{AD}$ patients have not been reported.

This study showed the diversity and abundance of plasma EVs collected from moderate-to-severe AD patients and healthy individuals via proteomic analysis and suggested that the candidates for uniquely or differentially expressed proteins in plasma EVs could be a diagnostic marker in AD.

\section{Materials and Methods Sample Collection}

Patients who met the following inclusion criteria were eligible: (1) patients age at least 18 years old or above with physician-diagnosed $\mathrm{AD},(2)$ according to the criteria of Williams, ${ }^{20}$ (3) having a SCORing Atopic Dermatitis (SCORAD) index ${ }^{21}$ of at least 25 or higher (moderate-tosevere). Exclusion criteria included patients with suspected or established primary immunodeficiency, patients receiving systemic corticosteroids, immunotherapeutic agents, and/or anti-infective drugs. Patients whose SCORAD score dropped below 25 (mild AD) were excluded.

Plasma samples were obtained from 12 patients with moderate-to-severe atopic dermatitis (AD) (8 males and 4 females, SCORAD range 30.6-72.9, mean 59.89 \pm 12.25 ) and 13 healthy subjects ( 7 males and 6 females). Each of the clinical subjects showed very severe AD symptoms causing them to visit the hospital for treatment. Healthy control (HC) subjects were screened through a general health examination. The experimental protocol was established, according to the ethical guidelines of the Helsinki Declaration and was approved by the Ethics Committee of Xiamen Chang Gung Hospital (Approval number XMCGIRB2020039). All patients and donors agreed to participate in this study and signed the informed consent.

\section{Isolation of EVs}

EVs were isolated from plasma samples by a series of ultracentrifugation (UC). Phosphate buffered saline (PBS) diluted the blood samples to decrease the viscosity. The diluted samples were centrifuged at $2000 \times \mathrm{g}$ for $30 \mathrm{~min}$ $\left(4^{\circ} \mathrm{C}\right)$ to eliminate the debris in the blood. The supernatant was removed from the new tubes, which were further centrifuged at $10,000 \times \mathrm{g}$ for $45 \mathrm{~min}\left(4^{\circ} \mathrm{C}\right)$. The supernatant was filtered with $0.45 \mu \mathrm{m}$ syringe filter (Millipore, USA) and the filtered supernatant was ultra-centrifuged at $100,000 \times \mathrm{g}$ for $70 \mathrm{~min}\left(4^{\circ} \mathrm{C}\right)($ Optima L-100XP, Beckman Coulter). The exosome pellet was resuspended with $10 \mathrm{~mL}$ cold PBS and repeated the ultracentrifugation step. The final exosome pellet was resuspended with $100 \mu \mathrm{L}$ of $0.22 \mu \mathrm{m}$-filtered PBS for subsequent analysis. 


\section{Sample Preparation}

The exosomes were lysed in $100 \mu \mathrm{L} 1 \%$ of Rapigest $(\mathrm{m} / \mathrm{v})$ in $50 \mathrm{mM}$ of ammonium bicarbonate. The lysate was initially reduced to $20 \mathrm{mM}$ of dithiothreitol (DTT) (Sigma) at $95^{\circ} \mathrm{C}$ for $5 \mathrm{~min}$, and subsequently alkylated in $50 \mathrm{mM}$ of iodoacetamide (IAA) (Sigma) for $30 \mathrm{~min}$ in the dark at room temperature (RT). After alkylation, the samples were centrifugated at $20000 \mathrm{~g}$ for $10 \mathrm{~min}$, the supernatant was transferred to a $10 \mathrm{kDa}$ centrifugal spin filter (Millipore) and sequentially washed with $200 \mu \mathrm{L}$ of $8 \mathrm{M}$ of urea for three times and $200 \mu \mathrm{L}$ of $50 \mathrm{mM}$ of ammonium bicarbonate for two times by centrifugation at 13500 g. Next, tryptic digestion was performed by adding trypsin (Promega) and Lys-C (Wako) at 1:50 (enzyme/ substrate, $\mathrm{m} / \mathrm{m}$ ) in $150 \mu \mathrm{L}$ of $50 \mathrm{mM}$ of ammonium bicarbonate at $37^{\circ} \mathrm{C}$ for $16 \mathrm{~h}$. Peptides were recovered by transferring the filter to a new collection tube and spinning at $13500 \mathrm{~g}$. To increase the yield of peptides, the filter was washed twice with $100 \mu \mathrm{L}$ of $50 \mathrm{mM}$ of ammonium bicarbonate. Peptides were desalted by StageTips and dried by speed vacuum at $45^{\circ} \mathrm{C}$.

\section{Transmission Electron Microscope (TEM)}

TEM with negative staining was used to observe the morphology of isolated exosomes. The purified exosome was diluted by two folds to $10 \mu \mathrm{L}$ and loaded onto a copper grid for $1 \mathrm{~min}$ and remove the excess exosome solution by filter paper carefully. The absorbed exosomes were stained with $10 \mu \mathrm{L} 2 \%$ uranyl acetate for $1 \mathrm{~min}$, and the excess fluid was removed with filter paper. Grids were dried for few minutes at room temperature and exosomal images were captured by TEM at $80 \mathrm{kV}$ (HT-7700, Hitachi HighTechnologies Corporation, Japan).

\section{Nano-Flow Cytometry Measurement (NFCM)}

The concentration and size of serum exosomes were analyzed by NFCM flow nano-Analyzer that following the manufacturer's instructions (N30E, nano-Analyzer, NanoFCM Inc., Xiamen, China). 200 nm Silica Nanosphere Cocktail was used to calibrate the concentration and size. $20 \mu \mathrm{L}$ from each sample was diluted by cold PBS (1:4 dilution). The $30 \mu \mathrm{L}$ diluted sample was single stained with $20 \mu \mathrm{L}$ Fluorescein Isothiocyanate (FITC) Mouse Anti-Human CD9 and FITC Mouse Anti-Human CD81 antibodies (BD Bioscience, USA) at $37^{\circ} \mathrm{C}$ for 30 minutes respectively. After incubation, the mixture was washed twice with PBS and centrifuged at $110,000 \times \mathrm{g}$ for $70 \mathrm{~min}$ at $4^{\circ} \mathrm{C}(\mathrm{CP} 100 \mathrm{MX}$, Hitachi High-Technologies Corporation, Japan). The supernatant was removed, and the pellet was resuspended with $50 \mu \mathrm{L}$ cold PBS. Events were recorded for 1 minute. The concentration, size distribution and surface proteins were quantified.

\section{Western Blot Analysis}

According to the manufacturer's instructions, protein concentrations of EVs were evaluated using a Bicinchoninic Acid (BCA) Protein Assay Kit (Beyotime, China). Bovine Serum Albumin (BSA) was used as the standard. Antibodies for EV markers of CD9 (Abcam, ab92726, 1:1000), TSG101 (Abcam, ab30870, 1:1000) and Calnexin (Abcam, ab22595, 1:1000) were tested using Western Blotting, using anti-mouse secondary antibody (BioLegend, 400108, 1:5000). 10 $\mu \mathrm{g}$ protein lysate was loaded onto $10 \%$ or $15 \%$ sodium dodecyl sulfate polyacrylamide gel electrophoresis (SDS-PAGE) gels dependent on the molecular weight of target proteins. The separated proteins were transferred to a methanol-activated polyvinylidene difluoride (PVDF) membrane (Merck Millipore, USA). The membrane in the blocking buffer containing $5 \%$ skim milk powder in $1 \mathrm{x}$ tris-buffered saline with tween 20 (TBST), for one hour at room temperature and then incubated overnight with the primary antibody at $4^{\circ} \mathrm{C}$. They were removing the primary antibodies by TBST three times of washing, following by another incubation with the secondary antibody for one hour at room temperature. Afterward, the membrane was incubated with an enhanced chemiluminescence (ECL) substrate (Merck Millipore, USA) for five minutes at room temperature, allowing for chemiluminescent signal capturing using the Chemiscope 3300 mini (Clinx, China).

\section{Liquid Chromatography with Tandem Mass Spectrometry (LC-MS/MS) Analysis} All MS experiments were performed on a nanoscale EASYnLC 1200UHPLC system (Thermo Fisher Scientific) connected to an Orbitrap Fusion Lumos equipped with a nanoelectrospray source (Thermo Fisher Scientific). Mobile phase A contained $0.1 \%$ of formic acid $(\mathrm{v} / \mathrm{v})$ in water; mobile phase B contained $0.1 \%$ of formic acid in $80 \%$ of acetonitrile (ACN). The peptides were dissolved in $0.1 \%$ of formic acid (FA) with $2 \%$ of acetonitrile and separated on a reversed-phase high-performance liquid chromatography (RP-HPLC) analytical column $(75 \mu \mathrm{m} \times 25 \mathrm{~cm})$ 
packed with $2 \mu \mathrm{m} \mathrm{C18}$ beads (Thermo Fisher Scientific) using a linear gradient ranging from $9 \%$ to $28 \%$ of $\mathrm{ACN}$ in $90 \mathrm{~min}$ and followed by a linear increase to $44 \% \mathrm{~B}$ in $20 \mathrm{~min}$ at a flow rate of $300 \mathrm{~nL} / \mathrm{min}$. The Orbitrap Fusion Lumos acquired data in a data-dependent manner alternating between full-scan MS and MS2 scans. The spray voltage was set at $2.2 \mathrm{kV}$ and the temperature of ion transfer capillary was $300^{\circ} \mathrm{C}$. The MS spectra $(350-1800 \mathrm{~m} / \mathrm{z})$ were collected with 120,000 resolution, automatic gain control (AGC) of $4 \times 10^{5}$, and $50 \mathrm{~ms}$ maximal injection time. Selected ions were sequentially fragmented in a 3 s cycle by high collision dissociation (HCD) with $30 \%$ normalized collision energy, specified isolated windows $1.6 \mathrm{~m} / \mathrm{z}, 15,000$ resolution. AGC of $5 \times 10^{4}$ and $40 \mathrm{~ms}$ maximal injection time were used. Dynamic exclusion was set to $30 \mathrm{~s}$. Unassigned ions or those with a charge of $1+$ and $>7+$ were rejected for MS/MS.

\section{Mass Spectrometry Data Analysis}

Raw data were processed using Proteome Discoverer (PD, version 2.2), and MS/MS spectra were searched against the reviewed SwissProt human proteome database. All searches were carried out with precursor mass tolerance of $20 \mathrm{ppm}$, fragment mass tolerance of $0.02 \mathrm{Da}$, oxidation (Met) (+15.9949Da) and acetylation (protein N-terminus) $(+42.0106 \mathrm{Da})$ as variable modifications, carbamidomethylation (Cys) (+57.0215Da) as fixed modification and three trypsin missed cleavages allowed. Only peptides with at least six amino acids in length were considered. The peptide and protein identifications were filtered by $\mathrm{PD}$ to control the false discovery rate (FDR) $<1 \%$. At least one unique peptide was required for protein identification.

\section{Functional and STRING Interaction Network Analysis}

Gene Ontology (GO) and Kyoto Encyclopaedia of Genes and Genomes (KEGG) pathway were analysed with the Database for Annotation, Visualization and Integrated Discovery (DAVID version 6.8) (https://david.ncifcrf. gov/). ${ }^{22}$ The biological process, molecular function and cellular component were further analysed using Panther software, online version (http://www.pantherdb.org/).

\section{Statistical Analysis}

Statistical analysis was performed using the Student's $t$-test to compare the plasma EVs proteins from patients and healthy controls. The p-value of 0.05 was considered statistically significant.

\section{Results}

\section{Demographic Characteristics of the Study Subjects}

A total of 25 subjects were enrolled that included 12 AD patients and 13 healthy controls. The difference in sex and age between the healthy control and patient groups was not significant $(\mathrm{P}=0.513$ and $\mathrm{P}=0.252$, respectively). Specific details are shown in Table 1.

\section{Isolation and Characterization of EVs}

Plasma EVs were isolated from $\mathrm{HC}$ and $\mathrm{AD}$ that were identified using TEM, nano-flow cytometer, and Western blot analysis. The characterization of typical cup-shaped vesicles was presented by TEM in EVs from the represented sample (Figure 1A). A nano-flow cytometer was used to measure the size and concentration of EVs. NFCM measurement revealed that the isolated EVs were 50-150 $\mathrm{nm}$ in size with an average size of $80.66 \pm 1.45 \mathrm{~nm}$ and $74.12 \pm 0.63 \mathrm{~nm}$ in $\mathrm{HC}$ and $\mathrm{AD}$ groups, respectively (Figure 1B). The EVs in the $\mathrm{HC}$ group were significantly larger in size compared to those in the $\mathrm{AD}$ group (Figure 1B). After normalization, particles concentration also was determined by $5.79 \times 10^{9} \pm 0.78 \times 10^{9} \mathrm{p} / \mathrm{mL}$ and $7.61 \times 10^{9} \pm 3.65 \times 10^{9} \mathrm{p} / \mathrm{mL}$ for $\mathrm{HC}$ and $\mathrm{AD}$, respectively. Although particle concentration of $\mathrm{AD}$ is higher slightly than those of $\mathrm{HC}$, the two groups are non-significantly different (Figure 1C). Western blotting was used to evaluate the purity of isolated EVs. Western blot analysis confirmed the presence of exosomal markers, such as CD9 and TSG101, whereas calnexin, as non-EV marker, was not detected (Figure 1D). Taken together, a series of centrifugation processes is successful to isolate EVs with purity and the amount of EVs is higher in AD patients.

Table I The Characteristics of Atopic Dermatitis Patients (AD) and Healthy Controls (HC)

\begin{tabular}{|l|l|l|l|}
\hline Variables & HC (n=13) & AD (n=12) & P value \\
\hline Sex (Male/Female) & $7 / 6(53.8 \%$ vs $46.2 \%)$ & $8 / 4(66.7 \%$ vs 33.3\%) & 0.513 \\
\hline Age (years) & $43.23 \pm 11.76$ & $52.33 \pm 25.15$ & 0.252 \\
\hline Total & $37.14 \pm 6.67$ & $59.75 \pm 22.66$ & 0.175 \\
\hline Male & $50.55 \pm 12.89$ & $37.50 \pm 26.10$ & 0.606 \\
\hline Female & - & $59.89 \pm 12.25$ & - \\
\hline SCORAD index & & & \\
\hline
\end{tabular}


A
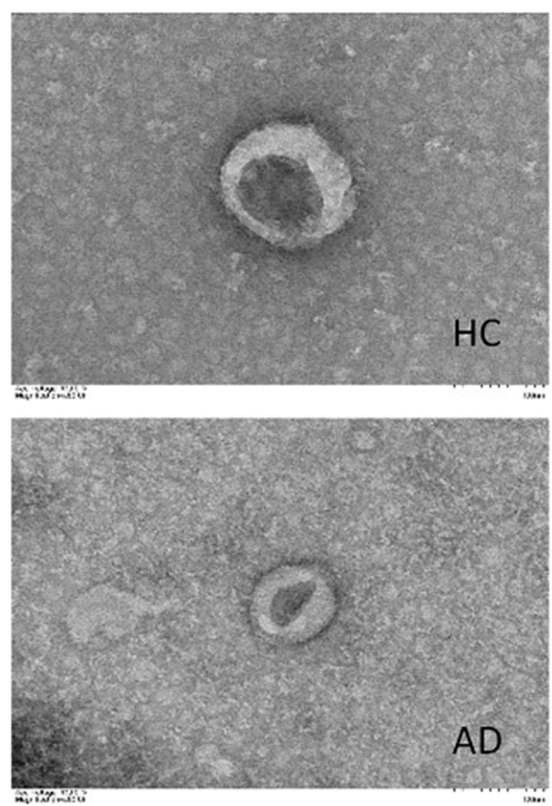

B
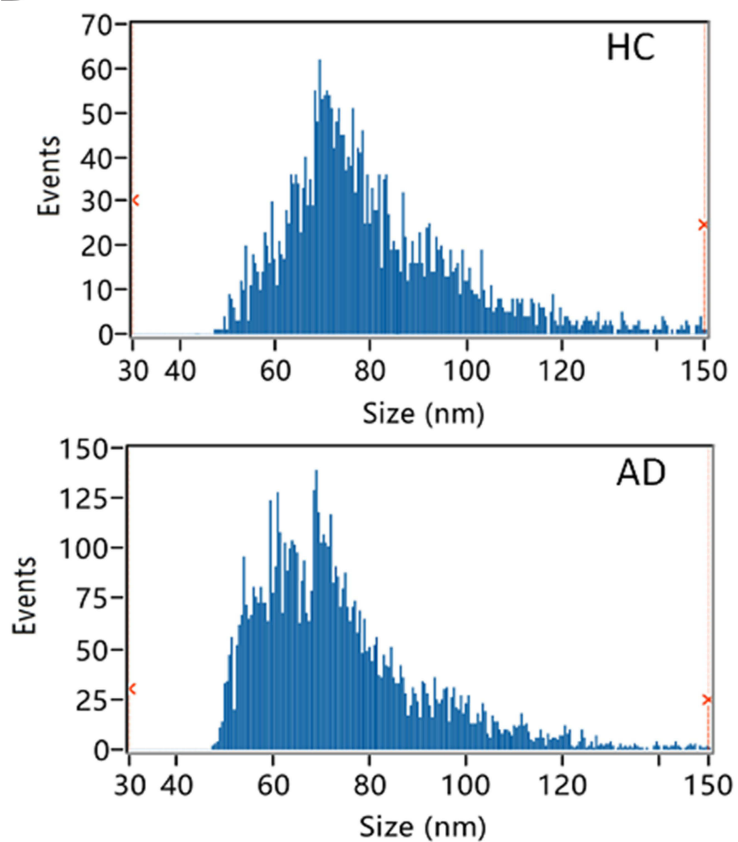

C
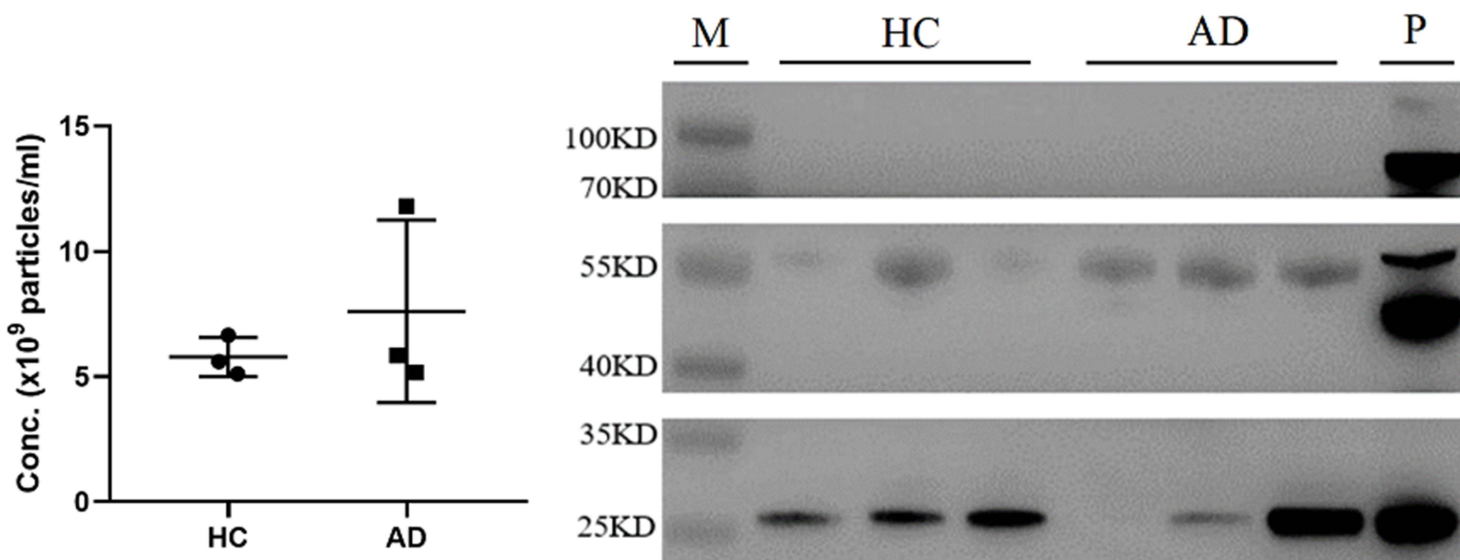

Calnexin

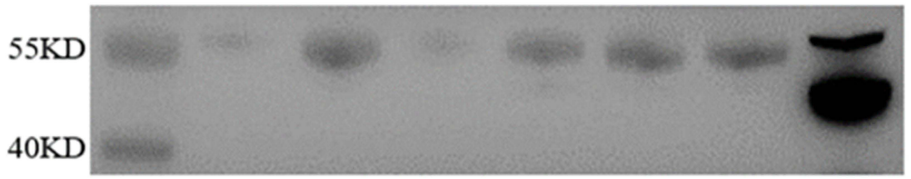

TSG101

$35 \mathrm{KD}$

$25 \mathrm{KD}$

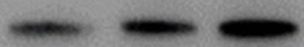

$\longrightarrow$ CD9

Figure I Isolation and identification of EVs from plasma. Electron micrographs of EVs were observed by transmission electron microscopy (bar=I00nm) (A); Nano-Flow Cytometry Measurement (NFCM) was used to detect the size (B) and concentration (C) of EVs; Western blot analysis to detect the expression of EV protein markers (CD9, TSGIOI) and one non-EV marker (Calnexin) (D).

Abbreviations: HC, healthy control group; AD, atopic dermatitis group.

\section{Proteomic Profiling of Plasma EVs}

Isolated EVs were analysed using the nano LC-MS/MS method to compare the plasma EVs proteins of patients with moderate-to-severe $\mathrm{AD}$ and $\mathrm{HC}$ patients. The proteomic analysis revealed a large number of proteins in plasma EVs from both $\mathrm{AD}$ and $\mathrm{HC}$ groups. A total of 1478 proteins in plasma EVs were found to be common proteins in $\mathrm{AD}$, whereas a total of 1597 proteins in plasma EVs were found to be common proteins in HC. A total of 1459 proteins in plasma EVs overlapped in comparing $\mathrm{HC}$ with AD (Figure 2). The volcano plot indicated the differential encapsulation of plasma EVs proteins between $A D$ and $\mathrm{HC}$ (Figure $3 \mathrm{~A}$ ). A total of 86 proteins in plasma EVs showed more than 2.5-fold up-regulation, while a total of 225 proteins in plasma EVs showed less than 1/2.5-fold down-regulation with a significant difference $(p<0.05)$ among AD compared with HC. The heat map showed 20 up-regulation proteins and 20 down-regulation proteins with a significant difference $(\mathrm{p}<0.05)$ in AD plasma EVs (Figure 3B and Table 2). 


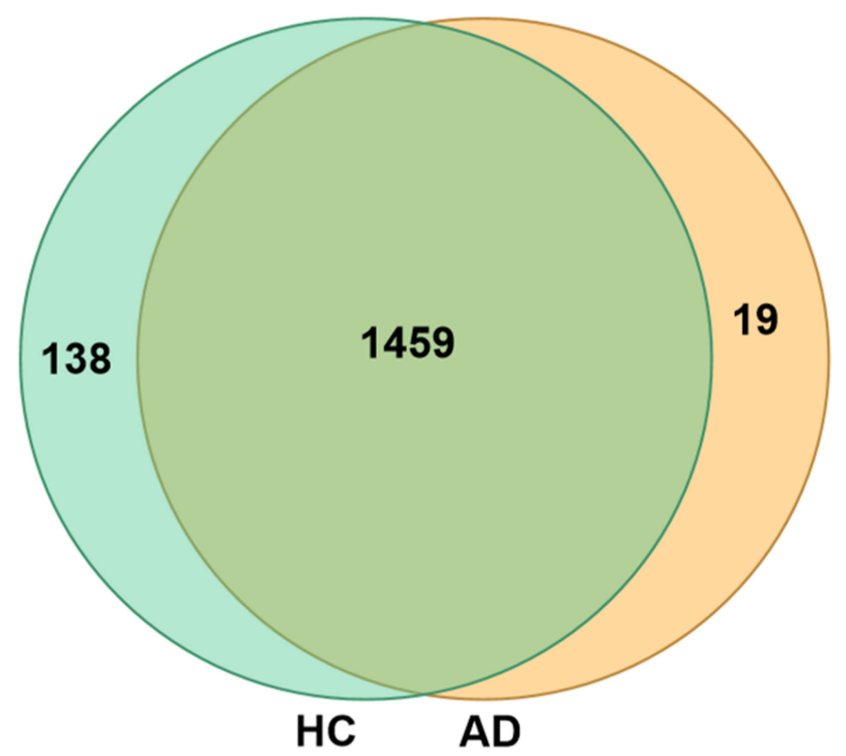

Figure 2 Comparative Venn diagram of plasma EVs proteins between $\mathrm{HC}$ group and $A D$ group.

\section{Functional and Protein-Protein Interaction (PPI) Network Analysis}

The GO analysis was performed to evaluate the candidates for differentially expressed proteins (Figure 4A-C) to obtain a comprehensive image of the proteins in plasma EVs from moderate-to-severe AD patients. The candidates for differentially expressed proteins in plasma EVs were interrogated for a broad range of biological processes, including localization, regulation of biological quality, single-multicellular organism process, cell activation, response to stimulus, and so on. In the molecular function category, most of the proteins appeared to participate in protein binding and structural molecular activity. Cellular component proteins were mainly localized in the extracellular membrane-bounded organelle, cytoplasm, and extracellular region.

The candidates for differentially expressed proteins in plasma EVs were illustrated in the protein-protein interaction (PPI) network by STRING analysis. Notably, the candidates for differentially expressed proteins in plasma EVs have been described a connectivity PPI network (Figure 5A) relating to several KEGG pathways, including pathways in platelet activation, leukocyte transendothelial migration, complement and coagulation cascades, phagosome, pathogenic Escherichia coli infection, Rap1 signaling pathway, and so on (top 10 KEGG pathways are shown in Figure 5B).

\section{Discussion}

It has been reported that EVs play an important role in cell-to-cell communication in normal physiology and

\section{A}

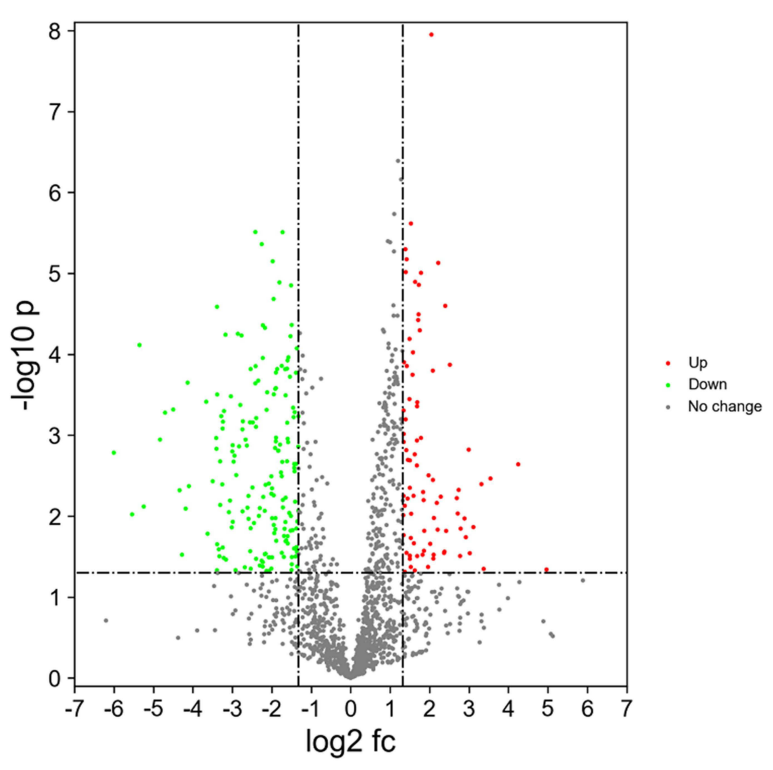

B

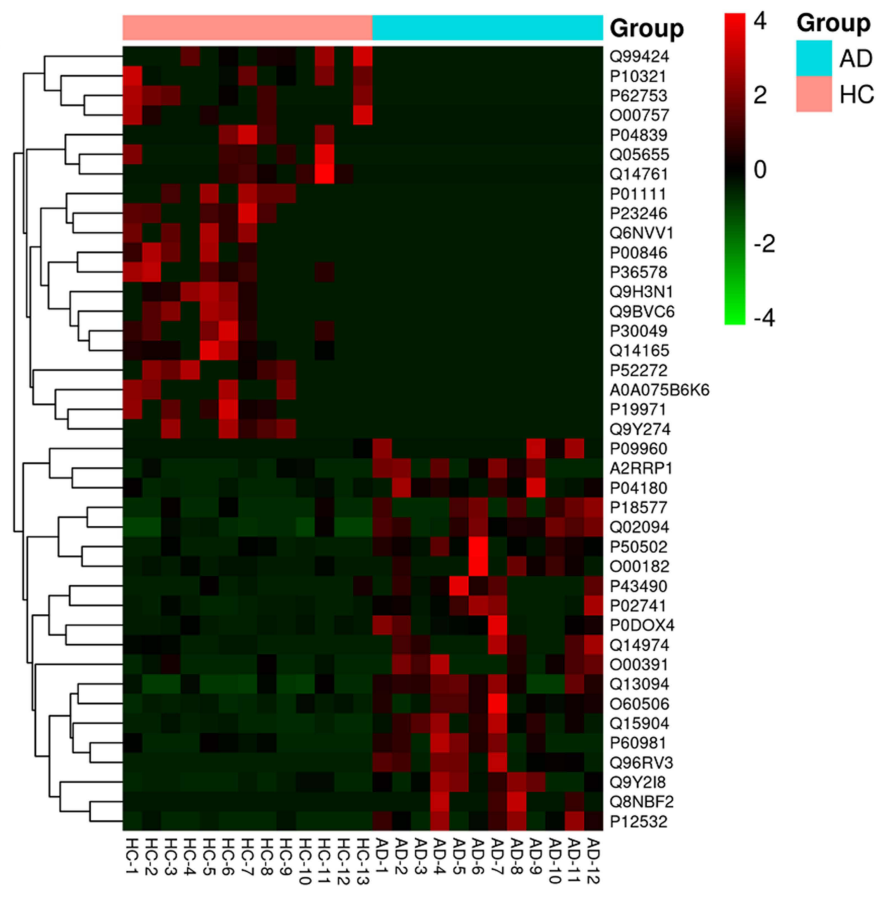

Figure 3 Volcano plot demonstrated the differential encapsulation of plasma EVs proteins between AD and HC. Up/down-regulated EVs proteins were indicated in green and red colors, respectively. Proteins not classified as up/down-regulated were plotted in grey color. X-axis and Y-axis indicate a more than two-fold change (in log2 scale) and $-\log 10$ with significance $(p<0.05)(\mathbf{A})$. Heat map of hierarchical clustering indicate the candidates for differentially expressed proteins in AD plasma EVs. Up/downregulated proteins are indicated by red/green color, respectively (B). GraphPad Prism software, version 8 (https://www.graphpad.com) was used to generate the volcano plot and heat map. 
Table 2 The Candidates for Differentially Expressed Proteins in Plasma EVs from AD Patients

\begin{tabular}{|c|c|c|c|}
\hline UniProt Accession & Protein Name & Gene Name & Regulation \\
\hline Q99424 & Peroxisomal acyl-coenzyme A oxidase 2 & ACOX2 & Down \\
\hline PI032I & HLA class I histocompatibility antigen, C alpha chain & HLA-C & Down \\
\hline P62753 & Ribosomal protein S6 & RPS6 & Down \\
\hline 000757 & Fructose-1,6-bisphosphatase isozyme 2 & FBP2 & Down \\
\hline P04839 & Cytochrome b- 245 heavy chain & CYBB & Down \\
\hline Q05655 & Protein kinase $C$ delta type & PRKCD & Down \\
\hline Q1476। & Protein tyrosine phosphatase receptor type C-associated protein & PTPRCAP & Down \\
\hline POIIII & GTPase NRas & NRAS & Down \\
\hline P23246 & Splicing factor, proline- and glutamine-rich & SFPQ & Down \\
\hline Q6NVVI & Ribosomal protein LI3a protein & RPLI3AP3 & Down \\
\hline P00846 & ATP synthase subunit alfa & MT-ATP6 & Down \\
\hline P36578 & Ribosomal protein L4 & RPL4 & Down \\
\hline Q9H3NI & Thioredoxin-related transmembrane protein I & TMXI & Down \\
\hline Q9BVC6 & Transmembrane protein 109 & TMEMI09 & Down \\
\hline P30049 & ATP synthase subunit delta & ATP5FID & Down \\
\hline Q14165 & Malectin & MLEC & Down \\
\hline P52272 & Heterogeneous nuclear ribonucleoprotein M & HNRNPM & Down \\
\hline A0A075B6K6 & Immunoglobulin lambda variable 4-3 & IGLV4-3 & Down \\
\hline PI997। & Thymidine phosphorylase & TYMP & Down \\
\hline Q9Y274 & Type 2 lactosamine alpha-2,3-sialyltransferase & ST3GAL6 & Down \\
\hline P09960 & Leukotriene A-4 hydrolase & LTA4H & Up \\
\hline A2RRPI & Neuroblastoma-amplified sequence & NBAS & Up \\
\hline P04I80 & Phosphatidylcholine-sterol acyltransferase & LCAT & $U_{p}$ \\
\hline PI8577 & Blood group Rh (CE) polypeptide & RHCE & Up \\
\hline Q02094 & Ammonium transporter $\mathrm{Rh}$ type $\mathrm{A}$ & RHAG & Up \\
\hline P50502 & Hsc70-interacting protein & STI3 & Up \\
\hline 000182 & Galectin-9 & LGALS9 & Up \\
\hline P43490 & Nicotinamide phosphoribosyl transferase & NAMPT & Up \\
\hline P0274I & C-reactive protein & CRP & Up \\
\hline PODOX4 & Immunoglobulin epsilon heavy chain & PODOX4 & Up \\
\hline Q14974 & Importin subunit beta-I & KPNBI & Up \\
\hline O00391 & Sulfhydryl oxidase I & QSOXI & Up \\
\hline Q13094 & Lymphocyte cytosolic protein 2 & LCP2 & Up \\
\hline O60506 & Heterogeneous nuclear ribonucleoprotein $\mathrm{Q}$ & SYNCRIP & Up \\
\hline Q15904 & V-type proton ATPase subunit SI & ATP6API & Up \\
\hline P6098I & Destrin & DSTN & Up \\
\hline Q96RV3 & Pecanex-like protein I & PCNXI & Up \\
\hline Q9Y218 & WD repeat-containing protein 37 & WDR37 & Up \\
\hline Q8NBF2 & $\mathrm{NHL}$ repeat-containing protein 2 & NHLRC2 & Up \\
\hline PI2532 & Creatine kinase U-type & CKMTIB & Up \\
\hline
\end{tabular}

pathological conditions. ${ }^{23}$ Wang et $\mathrm{al}^{12}$ reviewed the role of exosomes in inflammatory skin diseases and skin cancer, including systemic lupus erythematosus, psoriasis, atopic dermatitis, and melanoma. In conclusion, they indicated that EVs are involved in the onset or development of disease and may serve as diagnostic markers or predictive tools for prognosis and treatment responses. To date, increasing evidence has indicated that EVs are associated with the pathogenesis of AD. In this study, we compared the proteomic profiles of plasma-derived EVs between $\mathrm{AD}$ patients and $\mathrm{HC}$ patients for the first time. Proteomic analysis revealed a total of 1478 proteins in plasma EVs found as common proteins in $\mathrm{AD}$, whereas a total of 1597 proteins in plasma EVs were found to be common proteins in HC. From the result, a total of 19 proteins in plasma EVs from $\mathrm{AD}$ were the candidates for uniquely expressed proteins compared to those from HC. GO and KEGG analyses identified several functional terms that the 

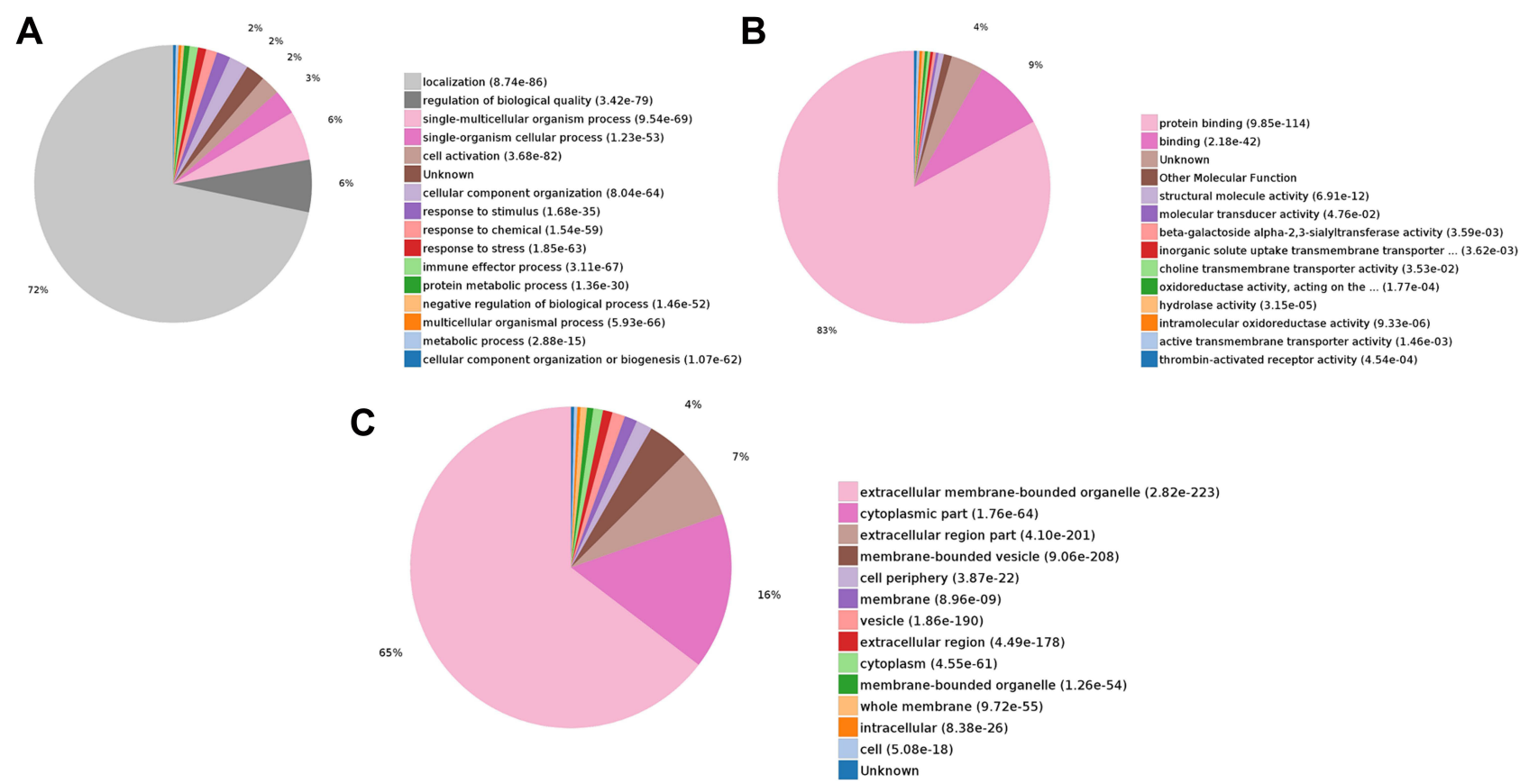

Figure $4 \mathrm{GO}$ analysis of the candidates for differentially expressed proteins in plasma EVs from AD. The candidates for differentially expressed proteins in plasma EVs from $A D$ were analysed using Panther software and categorised according to biological process (A), molecular function (B), cellular component (C).

candidates enriched for differentially expressed proteins in plasma EVs. Attention was paid to the aforementioned KEGG pathways.

The KEGG analysis revealed that the candidates for differentially expressed proteins were involved in many pathways, including pathways in platelet activation, leukocyte transendothelial migration, complement and coagulation cascades, phagosome, and so on. Thereby, the critical role of platelet activation has become apparent in inflammatory diseases. This encapsulates and describes all the procedures that platelets undergo to provide the necessary cofactors for the clotting system. ${ }^{24}$ As early as 2004, Kasperska-Zaj et al ${ }^{25}$ showed that patients with atopic eczema/dermatitis syndrome (AEDS) might have enhanced blood platelet activity as expressed by $\beta$-thrombomodulin $(\beta-\mathrm{TG})$ and platelet factor-4 (PF-4) level. A previous study also reported that elevated platelet activation markers, including $\beta$-TG, PF-4, vascular endothelial growth factor (VEGF) and platelet-derived growth factor (PDGF) in $\mathrm{AD}$ patients, are correlated with concomitant allergic diseases. ${ }^{26}$ Hilger et $\mathrm{al}^{27}$ demonstrated that platelet activation could enhance stimulation and responsiveness to Staphylococcus aureus, which is involved in AD pathogenesis. Taken together, platelet activation may contribute to the pathogenesis of $\mathrm{AD}$.
Our research showed that the proteins involved in platelet activation, including lymphocyte cytosolic protein 2 , and fibrinogen $\alpha / \beta / \gamma$ chain are upregulated. Among these proteins, lymphocyte cytosolic protein 2 (LCP2) has become a focus of our interest. LCP2, also called SLP-76 tyrosine phosphoprotein (SLP76), is expressed throughout the hematopoietic compartment. ${ }^{28}$ It is a key protein involved in TCR signaling and other hematopoietic pathways. ${ }^{29}$ Athari $^{30}$ confirmed that targeted therapy modulating cell signaling pathways, including high-affinity IgE receptor (Fc'RI)-LAT-SLP76, can be a powerful strategy to design new drugs to treat asthma. In addition, it has been reported that SLP76 is an essential signaling component to regulate FceRI-induced IL-4 production in basophils, ${ }^{31}$ while IL-4 provided by basophils plays a regulatory role in the initiation of Th2-type immune responses. ${ }^{32}$ So SLP76 may be involved in the pathogenesis of Th2-associated disease. However, whether it is associated with the occurrence and development of $\mathrm{AD}$ has yet to be established.

In conclusion, proteomic analysis identified a total of 1478 common proteins in plasma EVs from AD patients from which 19 proteins were candidates for uniquely expressed proteins compared with those of HC. Additionally, GO and KEGG analyses showed a new protein-SLP67 that may significantly contribute to $\mathrm{AD}$ pathogenesis. 

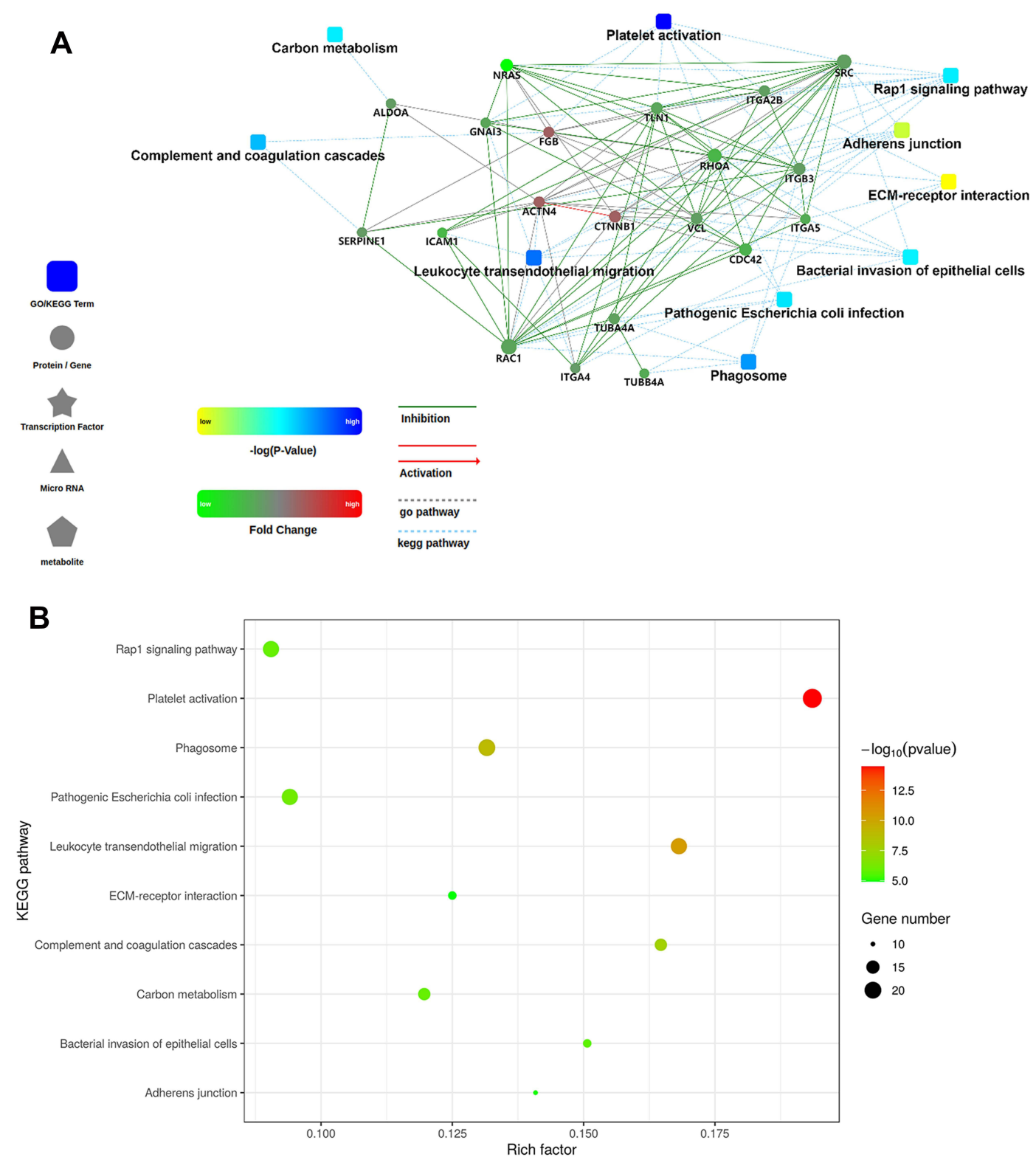

Figure 5 PPI network and KEGG pathway analysis. The PPI network was analysed by STRING software to evaluate the candidates for differentially expressed proteins in plasma EVs from AD (A). The molecular relationships are indicated by solid lines with or without arrow (Inhibitory/activating relations are indicated by green/red color, respectively). The gray or blue dotted lines represent GO pathway or KEEG pathway, respectively. The shape of square or circle separately stands for GO/KEGG term or protein/gene. Color set point is described in the left panel. The candidates for differentially expressed proteins in plasma EVs from AD were analysed using KEGG software, and the top 10 KEGG pathways were demonstrated in the bubble map (B). The node size reflects the number of gene. Color set point is described in the right panel.

This is the first study to present proteomic analysis of plasma EVs from AD patients. In the future, we will further verify the role of SLP67 in AD via animal and cell experiments to provide a promising therapeutic or diagnostic target.

\section{Acknowledgments}

We thank nurses and residents for their excellent assistance and careful handling of plasma samples, and all patients who participated in this study. We also thank
Xiamen LifeInt Technology Co., Ltd. for extracellular vesicle isolation. This work was supported by grants from the Natural Science Foundation of Fujian Province (No 2020J0142), Xiamen Chang Gung hospital and Xiamen Science and Technology Bureau, China (CMRPG1G0211, CMRPG1G0201).

\section{Funding}

This work was supported by grants from the Natural Science Foundation of Fujian Province (No 2020J0142), 
Xiamen Chang Gung hospital and Xiamen Science and Technology Bureau, China (CMRPG1G0211, CMRPG1 G0201).

\section{Disclosure}

The authors report no conflicts of interest in this work.

\section{References}

1. Gooderham MJ, Hong HC, Eshtiaghi P, Papp KA. Dupilumab: a review of its use in the treatment of atopic dermatitis. J Am Acad Dermatol. 2018;78 (3 Suppl 1):S28-s36. doi:10.1016/j.jaad.2017.12.022

2. Langan SM, Irvine AD, Weidinger S. Atopic dermatitis. Lancet. 2020;396(10247):345-360. doi:10.1016/S0140-6736(20)31286-1

3. Silverberg JI. Comorbidities and the impact of atopic dermatitis. Ann Allergy Asthma Immunol. 2019;123(2):144-151. doi:10.1016/j.anai.20 19.04.020

4. Sacotte R, Silverberg JI. Epidemiology of adult atopic dermatitis. Clin Dermatol. 2018;36(5):595-605. doi:10.1016/j.clindermatol.2018.05.007

5. Garg N, Silverberg JI. Epidemiology of childhood atopic dermatitis. Clin Dermatol. 2015;33(3):281-288. doi:10.1016/j.clindermatol.2014.12.004

6. van Niel G, D'Angelo G, Raposo G. Shedding light on the cell biology of extracellular vesicles. Nat Rev Mol Cell Biol. 2018;19 (4):213-228. doi:10.1038/nrm.2017.125

7. Vega VL, Rodríguez-Silva M, Frey T, et al. Hsp70 translocates into the plasma membrane after stress and is released into the extracellular environment in a membrane-associated form that activates macrophages. J Immunol. 2008;180(6):4299-4307. doi:10.4049/ jimmunol.180.6.4299

8. Zhao H, Yang L, Baddour J, et al. Tumor microenvironment derived exosomes pleiotropically modulate cancer cell metabolism. eLife. 2016;5:e10250. doi:10.7554/eLife.10250

9. Asai H, Ikezu S, Tsunoda S, et al. Depletion of microglia and inhibition of exosome synthesis halt tau propagation. Nat Neurosci. 2015;18(11):1584-1593. doi:10.1038/nn.4132

10. Dreux M, Garaigorta U, Boyd B, et al. Short-range exosomal transfer of viral RNA from infected cells to plasmacytoid dendritic cells triggers innate immunity. Cell Host Microbe. 2012;12(4):558-570. doi: $10.1016 /$ j.chom. 2012.08 .010

11. He C, Zheng S, Luo Y, Wang B. Exosome theranostics: biology and translational medicine. Theranostics. 2018;8(1):237-255. doi:10.71 50/thno. 21945

12. Wang WM, Wu C, Jin HZ. Exosomes in chronic inflammatory skin diseases and skin tumors. Exp Dermatol. 2019;28(3):213-218.

13. Aslam B, Basit M, Nisar MA, Khurshid M, Rasool MH. Proteomics: technologies and their applications. J Chromatogr Sci. 2017;55 (2):182-196. doi:10.1093/chromsci/bmw167

14. Domon B, Aebersold R. Mass spectrometry and protein analysis. Science. 2006;312(5771):212-217. doi:10.1126/science.1124619

15. Koba T, Takeda Y, Narumi R, et al. Proteomics of serum extracellular vesicles identifies a novel COPD biomarker, fibulin-3 from elastic fibres. ERJ Open Res. 2021;7(1):00658-2020. doi:10.1183/2312 0541.00658-2020

16. Shin KO, Ha DH, Kim JO, et al. Exosomes from human adipose tissue-derived mesenchymal stem cells promote epidermal barrier repair by inducing de novo synthesis of ceramides in atopic dermatitis. Cells. 2020;9(3):680. doi:10.3390/cells9030680
17. Cho BS, Kim JO, Ha DH, Yi YW. Exosomes derived from human adipose tissue-derived mesenchymal stem cells alleviate atopic dermatitis. Stem Cell Res Ther. 2018;9(1):187. doi:10.1186/s13287018-0939-5

18. Hong SW, Kim MR, Lee EY, et al. Extracellular vesicles derived from Staphylococcus aureus induce atopic dermatitis-like skin inflammation. Allergy. 2011;66(3):351-359. doi:10.1111/j.13989995.2010.02483.x

19. Yang J, McDowell A, Seo H, et al. Diagnostic models for atopic dermatitis based on serum microbial extracellular vesicle metagenomic analysis: a Pilot Study. Allergy Asthma Immunol Res. 2020;12(5):792-805. doi:10.4168/aair.2020.12.5.792

20. Williams HC, Burney PG, Hay RJ, et al. The U.K. Working Party's Diagnostic Criteria for Atopic Dermatitis. I. Derivation of a minimum set of discriminators for atopic dermatitis. $\mathrm{Br}$ J Dermatol. 1994;131(3):383-396. doi:10.1111/j.1365-2133.1994. tb08530.x

21. Kunz B, Oranje AP, Labreze L, Stalder JF, Ring J, Taieb A. Clinical validation and guidelines for the SCORAD index: consensus report of the European Task Force on Atopic Dermatitis. Dermatology. 1997;195(1):10-19. doi:10.1159/000245677

22. Sherman BT, Lempicki RA. Systematic and integrative analysis of large gene lists using DAVID bioinformatics resources. Nat Protoc. 2009;4(1):44-57. doi:10.1038/nprot.2008.211

23. Todorova D, Simoncini S, Lacroix R, Sabatier F, Dignat-George F. Extracellular vesicles in angiogenesis. Circ Res. 2017;120 (10):1658-1673. doi:10.1161/CIRCRESAHA.117.309681

24. Rubenstein DA, Yin W. Platelet-activation mechanisms and vascular remodeling. Compr Physiol. 2018;8(3):1117-1156.

25. Kasperska-Zaj A, Nowakowski M, Rogala B. Enhanced platelet activation in patients with atopic eczema/dermatitis syndrome. Inflammation. 2004;28(5):299-302. doi:10.1007/s10753-004-60 54-z

26. Nastałek M, Potaczek DP, Wojas-Pelc A, Undas A. Plasma platelet activation markers in patients with atopic dermatitis and concomitant allergic diseases. J Dermatol Sci. 2011;64(1):79-82. doi:10.1016/j. jdermsci.2011.07.001

27. Hilger RA, Neuber K, König W. Conversion of leukotriene A4 by neutrophils and platelets from patients with atopic dermatitis. Immunology. 1991;74(4):689-695.

28. Koretzky GA, Abtahian F, Silverman MA. SLP76 and SLP65: complex regulation of signalling in lymphocytes and beyond. Nat Rev Immunol. 2006;6(1):67-78. doi:10.1038/nri1750

29. Lev A, Lee YN, Sun G, et al. Inherited SLP76 deficiency in humans causes severe combined immunodeficiency, neutrophil and platelet defects. J Exp Med. 2021;218(3). doi:10.1084/jem.20201062

30. Athari SS. Targeting cell signaling in allergic asthma. Signal Transduct Target Ther. 2019;4:45.

31. Hidano S, Kitamura D, Kumar L, Geha RS, Goitsuka R. SLP-76 is required for high-affinity IgE receptor- and IL-3 receptor-mediated activation of basophils. Int Immunol. 2012;24(11):719-727. doi:10.1093/intimm/dxs072

32. Yoshimoto T, Yasuda K, Tanaka H, et al. Basophils contribute to $\mathrm{T}(\mathrm{H}) 2-\mathrm{IgE}$ responses in vivo via IL-4 production and presentation of peptide-MHC class II complexes to CD4+ T cells. Nat Immunol. 2009;10(7):706-712. doi:10.1038/ni.1737 


\section{Publish your work in this journal}

Clinical, Cosmetic and Investigational Dermatology is an international, peer-reviewed, open access, online journal that focuses on the latest clinical and experimental research in all aspects of skin disease and cosmetic interventions. This journal is indexed on CAS.

The manuscript management system is completely online and includes a very quick and fair peer-review system, which is all easy to use. Visit http://www.dovepress.com/testimonials.php to read real to use. Visit http://www.dovepress.
quotes from published authors.

Submit your manuscript here: https://www.dovepress.com/clinical-cosmetic-and-investigational-dermatology-journal 